\title{
Intoxication aiguë au dioxyde de carbone
}

\author{
Clinical Case of an Acute Carbon Dioxide Poisoning
}

\author{
A. Mastrodicasa $\cdot$ A. Cuenoud $\cdot$ M. Pasquier $\cdot$ P.-N. Carron
}

Reçu le 1 juin 2018 ; accepté le 30 juillet 2018

(C) SFMU et Lavoisier SAS 2018

\section{Introduction}

Il y a 2200 ans, dans le sanctuaire romain de Hiérapolis dédié à Pluton, des rites impliquant des sacrifices d'animaux étaient régulièrement pratiqués. De jeunes taureaux mourraient ainsi mystérieusement en approchant de « la porte des enfers ». Les prêtres du culte de Cybèle qui les accompagnaient échappaient quant à eux à la mort. Dans l'Antiquité, on les pensait protégés du souffle de Cerbère par la castration. De récentes recherches ont révélé plus prosaïquement la présence de taux élevés de dioxyde de carbone $\left(\mathrm{CO}_{2}\right)$, liés à l'activité volcanique des lieux [1] Les prêtres se protégeaient vraisemblablement en se maintenant en hauteur et en choisissant les heures du jour les plus propices [1].

Le $\mathrm{CO}_{2}$ est un gaz normalement présent à très faible concentration dans l'air ambiant, mais pouvant se retrouver en quantité supraphysiologique dans certaines conditions environnementales, accidentelles ou professionnelles. À doses modérées, le $\mathrm{CO}_{2}$ produit des symptômes aspécifiques, mais s'avère rapidement létal à des concentrations élevées. Bien que des situations d'intoxications soient régulièrement rapportées en Europe, le risque de toxicité lié au $\mathrm{CO}_{2}$ et les principes de sa prise en charge sont peu connus des urgentistes.

A. Mastrodicasa

Service des urgences, université de Liège,

B-4000 Liège, Belgique

A. Cuenoud

Service d'anesthésiologie,

centre hospitalier universitaire Vaudois,

université de Lausanne, CH-1011 Lausanne, Suisse

M. Pasquier · P.-N. Carron $(\varangle)$

Service des urgences,

centre hospitalier universitaire Vaudois,

université de Lausanne, , CH-1011 Lausanne, Suisse

e-mail : pierre-nicolas.carron@chuv.ch

\section{Observation}

Fin octobre, vers 13 h 50, un appel est transmis à la centrale d'appels d'urgences, annonçant un accident dans une exploitation agricole avec deux personnes inconscientes. Les premières informations à disposition des secours confirment un incident survenu dans un silo de stockage de pulpe de betterave. Trois patients sont identifiés, présentant à des degrés divers des troubles de l'état de conscience :

- un jeune apprenti de 17 ans avec une perte de connaissance prolongée, précédée d'une dyspnée sévère et de vertiges. Le patient est tombé de sa hauteur dans un fond du silo, avec la tête en partie immergée dans de l'eau de pluie. En raison d'un tableau clinique évocateur d'un arrêt cardiorespiratoire (cyanose, absence de respiration, mouvements toniques des membres supérieurs), il a bénéficié d'un massage cardiaque externe par les témoins pendant quelques minutes, avec reprise de conscience rapide ;

- une jeune femme de 25 ans, enceinte de trois mois, sortie seule du silo après avoir présenté une syncope ;

- un homme de 30 ans ayant également présenté une brève perte de connaissance. Deux autres hommes, âgés d'une trentaine d'années, ont été brièvement exposés au même environnement, mais ne présentent aucun symptôme. Ils ont rapidement procédé à l'aération du silo, pratiqué les premiers secours et extrait les patients. La prise en charge sur site par le Smur s'est limitée à une oxygénation à haut débit et à la pose d'un accès vasculaire avec hydratation IV. La présence de $\mathrm{CO}_{2}$ en forte concentration est identifiée par les pompiers sur site.

Aux urgences, le jeune apprenti est conscient, tachypnéique à $40 /$ minute, avec une acidose respiratoire $(\mathrm{pH}$ : 7,$15 ; \mathrm{pCO}_{2}$ artérielle à $75 \mathrm{mmHg}$; bicarbonates : $27 \mathrm{mmol} / \mathrm{l}$ ) et une $\mathrm{SpO}_{2}$ à $99 \%$ sous une $\mathrm{FiO}_{2}$ de $100 \%$. En raison de la suspicion de submersion et d'intoxication concomitante au $\mathrm{CO}_{2}$, il est intubé et ventilé mécaniquement durant 36 heures. L'ECG ainsi qu'une échocardiographie sont dans les normes. À un mois, l'évolution est favorable, et le diagnostic différentiel 
perdure entre une syncope ou un épisode de convulsions, voire un éventuel arrêt cardiorespiratoire.

La patiente enceinte est hospitalisée durant 48 heures et bénéficie également d'un bilan cardiologique. Une élévation isolée des valeurs de troponines ultrasensibles (pic : $239 \mathrm{ng} / \mathrm{l}$ pour une norme $<14 \mathrm{ng} / \mathrm{l}$ ) et une discrète hypokinésie septoinféro-basale asymptomatique sont attribuées à l'intoxication aiguë. L'examen obstétrical ne révèle pas d'anomalie. Lors d'un contrôle, un mois plus tard, la patiente est asymptomatique, et l'échocardiographie est dans les normes. Les autres patients impliqués ont bénéficié également d'une brève surveillance, avec une évolution clinique sans particularité.

\section{Propriétés physicochimiques et physiologiques du $\mathrm{CO}_{2}$}

À température et pression standard, le $\mathrm{CO}_{2}$ est un gaz non inflammable, stable, sans couleur ni odeur franche. Sa densité relative plus importante par rapport à l'air $(1,52)$ favorise sa distribution préférentielle dans les parties inférieures des bâtiments. L'air extérieur est constitué en teneur volumique à $78,084 \%$ d'azote, $20,946 \%$ d'oxygène et à $0,934 \%$ d'argon. Le reste est composé de gaz mineurs, dont le plus important proportionnellement est le $\mathrm{CO}_{2}$ avec 380 parties par million (ppm) [2]. L'air contenu à l'intérieur des bâtiments comprend, quant à lui, en moyenne 0,08 à $0,1 \%$ de $\mathrm{CO}_{2}$ [2].

Physiologiquement, le $\mathrm{CO}_{2}$ est un produit du métabolisme cellulaire. Au niveau mitochondrial, la respiration cellulaire produit par oxydation complète d'une mole de glucose $\left(\mathrm{C}_{6} \mathrm{H}_{12} \mathrm{O}_{6}\right)$ avec 6 moles d'oxygène, de l'énergie, 6 moles de $\mathrm{CO}_{2}$ et 6 moles d'eau. Pour un homme au repos, le métabolisme génère ainsi environ $200 \mathrm{ml} \mathrm{de} \mathrm{CO}_{2}$ par minute. $\mathrm{Le} \mathrm{CO}_{2}$ produit par le métabolisme est transporté sous forme de bicarbonate ( $87 \%$ ), de composés liés aux protéines du plasma ( $7 \%$, principalement l'hémoglobine), d'acide carbonique $(<1 \%)$ ou en solution $(6 \%)$ [3]. La solubilité du $\mathrm{CO}_{2}$ est 30 fois supérieure à celle de l'oxygène et sa diffusion 20 fois plus importante. $\mathrm{Le} \mathrm{CO}_{2}$ est finement régulé au niveau de l'organisme avec le $\mathrm{pH}$, afin que celui-ci reste relativement constant. Toute activité (endogène ou exogène) majorant le $\mathrm{CO}_{2}$ diminue le $\mathrm{pH}$ et stimule le centre respiratoire au niveau du tronc cérébral. Le corps peut compenser l'acidose plasmatique en augmentant sa concentration en bicarbonates, dans un premier temps (en heures) par les cellules, et dans un second temps (en jours) par une réabsorption rénale [4]. Cette adaptation est lente et ne permet pas de répondre à une élévation importante et rapide de $\mathrm{CO}_{2}$ exogène.

Le $\mathrm{CO}_{2}$ joue également un rôle au niveau des échanges gazeux à travers l'effet Bohr et l'effet Haldane. L'effet Bohr est la diminution de l'affinité de l'hémoglobine pour l'oxy- gène lors d'une augmentation de la $\mathrm{PCO}_{2}$ ou d'une diminution $\mathrm{du} \mathrm{pH}$, favorisant une libération accrue d'oxygène dans les régions riches en $\mathrm{CO}_{2}$ ou en acides, comme par exemple les muscles en condition d'effort physique. À l'opposé, l'effet Haldane favorise une réduction de l'affinité de l'hémoglobine pour le $\mathrm{CO}_{2}$ lors d'une augmentation de la pression partielle en $\mathrm{O}_{2}$, classiquement au niveau pulmonaire, facilitant dès lors une réoxygénation du sang.

\section{Toxicité aiguë}

La toxicité du $\mathrm{CO}_{2}$ est liée à deux mécanismes distincts :

- une asphyxie cellulaire, provoquée par l'hypoxie relative ;

- une toxicité cellulaire directe, indépendante de l'hypoxie $[5,6]$.

Dans une atmosphère riche en $\mathrm{CO}_{2}$, le patient inhale ainsi une fraction inspirée en $\mathrm{CO}_{2}$ accrue et une fraction en $\mathrm{O}_{2}$ plus faible. Cette situation favorise un état hypoxique, également favorisé par la réduction de l'affinité de l'hémoglobine pour l'oxygène (cf. supra). De par sa grande solubilité et son libre passage au travers des membres lipidiques cellulaires, le $\mathrm{CO}_{2}$ diffuse rapidement au travers des tissus. Son augmentation de concentration majore la production d'ions $\mathrm{H}+$ et favorise l'apparition d'une acidose. Cette acidose stimule initialement les chémorécepteurs au niveau des centres respiratoires du bulbe, induisant une augmentation du volume courant, puis une majoration de la fréquence respiratoire. L'augmentation de $\mathrm{CO}_{2}$ devient ainsi symptomatique sur le plan ventilatoire à partir de 3-4\% de $\mathrm{CO}_{2}$ dans l'air inspiré, avec de grandes variations interindividuelles [7]. L'élévation du $\mathrm{CO}_{2}$ augmente également la fréquence ainsi que le débit cardiaque, augmente la pression artérielle et provoque une vasodilatation périphérique $[6,8]$. Au niveau cérébral, le $\mathrm{CO}_{2}$ possède une puissante action vasodilatatrice. À des concentrations sanguines de 5-7\% de $\mathrm{CO}_{2}$, le débit sanguin cérébral augmente de $75 \%$, ce qui favorise un apport de sang avec une $\mathrm{PCO}_{2}$ élevée, aggravant la toxicité cérébrale [9].

S'il exerce initialement un effet stimulant sur le système nerveux central, le $\mathrm{CO}_{2}$ possède à des taux supérieurs à 10 $15 \%$ des effets cérébraux dépresseurs [9]. On peut rappeler à cet effet que le $\mathrm{CO}_{2}$ a été utilisé dans le passé comme anesthésique général, mais abandonné en raison des effets secondaires et en particulier du risque de convulsions associées. In fine, l'intoxication au $\mathrm{CO}_{2}$ conduit à une réduction du tonus circulatoire et respiratoire, favorisant tant le cercle vicieux de l'acidose respiratoire que le collapsus circulatoire. Si l'acidose se poursuit, elle devient létale par entrave des réactions enzymatiques des voies énergétiques $[6,8]$.

Le $\mathrm{CO}_{2}$ génère également des effets toxiques proinflammatoires, en particulier lors d'exposition chronique [10]. À un taux d'oxygène constant de $21 \%$, une majoration 
de la teneur en $\mathrm{CO}_{2}$ supérieure à $5 \%$ favorise ainsi la sécrétion, dose-dépendante, de cytokines pro-inflammatoires (IL-6, IL-8, TNF-alpha) [10,11].

\section{Présentation clinique}

Le tableau 1 résume la symptomatologie et la réponse physiopathologique selon la teneur en $\mathrm{CO}_{2}$ dans l'air inspiré. Les symptômes sont généralement dose-dépendants et augmentent avec la durée d'exposition $[8,9,12]$. Il existe néanmoins une grande variabilité de tolérance au $\mathrm{CO}_{2}$, expliquant les larges intervalles de concentration par rapport aux symptômes et l'absence dès lors de cut-off précis. Cette tolérance

\begin{tabular}{|c|c|c|}
\hline $\begin{array}{l}\text { Teneur en } \mathrm{CO}_{2} \\
\text { en } \% \\
\text { volumique }\end{array}$ & $\begin{array}{l}\text { En parties } \\
\text { par million } \\
(\text { ppm) }\end{array}$ & Symptomatologie \\
\hline 0,03 & 300 & $\begin{array}{l}\text { Absence de symptômes } \\
\text { (air ambiant usuel) }\end{array}$ \\
\hline $0,3-0,5$ & $3000-5000$ & Céphalées éventuelles \\
\hline 0,5 & 5000 & $\begin{array}{l}\text { Augmentation de } 5 \% \\
\text { de la ventilation }\end{array}$ \\
\hline 1 & 10000 & $\begin{array}{l}\text { Inattention, asthénie, } \\
\text { faiblesse }\end{array}$ \\
\hline 2 & 20000 & $\begin{array}{l}\text { Augmentation de } 50 \% \\
\text { de la ventilation }\end{array}$ \\
\hline 4 & 40000 & $\begin{array}{l}\text { Seuil de danger immédiat } \\
\text { requérant une évacuation } \\
\text { rapide }\end{array}$ \\
\hline $5-7$ & $50000-70000$ & $\begin{array}{l}\text { Augmentation du flux } \\
\text { sanguin cérébral, troubles } \\
\text { de la concentration, } \\
\text { troubles visuels, } \\
\text { paresthésies, céphalées, } \\
\text { anxiété, bouffées } \\
\text { de chaleur } \\
\text { Palpitations, tachycardie } \\
\text { progressive, hypertension } \\
\text { systolique et diastolique }\end{array}$ \\
\hline $5-10$ & $50000-100000$ & $\begin{array}{l}\text { Dyspnée sévère, acidose } \\
\text { respiratoire, arythmies }\end{array}$ \\
\hline 10 & 100000 & $\begin{array}{l}\text { Perte de conscience } \\
\text { rapide }\end{array}$ \\
\hline 15 & 150000 & $\begin{array}{l}\text { Perte de conscience } \\
\text { subite }\end{array}$ \\
\hline 20 & 200000 & Clonies, apnée \\
\hline $30-40$ & $\begin{array}{l}300000- \\
400000\end{array}$ & Décès potentiel \\
\hline
\end{tabular}

diminue avec l'âge et concerne plus particulièrement les fumeurs en raison de l'inhalation chronique de grandes quantités de $\mathrm{CO}_{2}[10,12]$. L'intoxication au $\mathrm{CO}_{2}$ est fréquemment confondue avec celle en lien avec le monoxyde de carbone (CO). Si une partie de la physiopathologie est similaire, il existe néanmoins des différences importantes, tant dans le mécanisme toxique que dans la prise en charge (Tableau 2).

\section{Circonstances et contexte d'utilisation du $\mathrm{CO}_{2}$}

Une production de $\mathrm{CO}_{2}$ est observée dans diverses situations professionnelles ou lors de combustion de dérivés du carbone, lors de fermentations alcooliques ou lactiques, au départ de sources telluriques, ou lors de production endogène en milieu confiné (Tableau 3) [8].

L'utilisation professionnelle de $\mathrm{CO}_{2}$ est fréquente dans la production agroalimentaire. Le $\mathrm{CO}_{2}$ sert d'accélérateur de croissance dans les serres des cultures maraîchères, d'agent d'extinction gazeux sur les sites à haut risque d'incendie, pour la carbonatation de boissons gazeuses, ou pour la réfrigération et surgélation de produits alimentaires (neige carbonique) [13]. Des accidents peuvent survenir en cas de fuite dans des milieux clos mal ventilés (caves, ascenseurs, camions réfrigérés) et/ou en cas de défaillances de détecteurs de $\mathrm{CO}_{2}$ dans les usines. Du $\mathrm{CO}_{2}$ est également utilisé lors de procédés d'extraction de l'aluminium, dans certaines méthodes d'euthanasie animalière, ou comme facteur de croissance de milieux de culture [9].

$\mathrm{Du} \mathrm{CO}_{2}$ est enfin produit lors des processus de fermentation ; durant les étapes de production (boissons alcooliques, boulangerie), de stockage (silo, cale de bateau) ou de remise en état des locaux (cuves de vendanges, fosses, égouts). À ce titre, le silo à fourrage constitue un risque particulier. L'ensilage est une technique de conservation utilisant la fermentation lactique en milieu humide. Ce mode de conservation s'applique à différents fourrages pour le bétail (maïs, betterave, etc.). La fermentation permet de rendre le fourrage plus digeste et nourrissant, tout en garantissant une conservation plus longue, l'abaissement du pH inhibant le développement de moisissures, levures ou bactéries.

Les sources telluriques impliquent les sous-sols des grottes et cavernes, les mines, mais aussi les volcans, les lacs volcaniques et les émissions géothermiques de gaz. Ces sources de $\mathrm{CO}_{2}$ ne sont pas anodines, comme en témoigne la catastrophe du lac Nyos, survenue en 1986 au Cameroun, avec 1700 décès par intoxication au $\mathrm{CO}_{2}$ dans un périmètre de $20 \mathrm{~km}$ [14].

Finalement, la production de $\mathrm{CO}_{2}$ par le métabolisme physiologique peut présenter un risque lorsqu'elle survient en milieux confinés, tels que scaphandres, sous-marins, 
Tableau 2 Tableau comparatif entre monoxyde et dioxyde de carbone

\begin{tabular}{|lll|}
\hline & Monoxyde de carbone $\mathbf{( C O )}$ & Dioxyde de carbone $\left(\mathbf{C O} \mathbf{O}_{\mathbf{2}}\right)$ \\
\hline $\begin{array}{l}\text { Type de gaz } \\
\text { Localisation }\end{array}$ & Gaz inodore et incolore & Gaz inodore et incolore \\
Létalité & Espace clos & Espace clos \\
Symptomatologie & Mortel à haute concentration $(700 \mathrm{ppm})$ & Mortel à haute concentration $(80000 \mathrm{ppm})$ \\
& Céphalées, vertiges et autres symptômes & Céphalées, vertiges et autres symptômes \\
Origine & aspécifiques à moins de 100 ppm & aspécifiques à moins de 30000 ppm \\
& Intoxication résultant d'une mauvaise combustion & Présence naturelle, mais intoxication également \\
Téguments & & industrielle \\
Potentiel inflammable & Cyanose/conjonctives rosées & Pas de cyanose ou d'érythrose \\
Effet sur l'hémoglobine & Formation de carboxyhémoglobine (HbCO) & Non inflammable \\
Fréquence des intoxications & Élevée & Absence de carboxyhémoglobine (HbCO) \\
\hline
\end{tabular}

\begin{tabular}{|c|c|}
\hline $\begin{array}{l}\text { Domaine } \\
\text { agroalimentaire }\end{array}$ & $\begin{array}{l}\text { Accélérateur de croissance } \\
\text { pour les cultures maraîchères, } \\
\text { carbonatation de boissons gazeuses, } \\
\text { réfrigération et surgélation } \\
\text { de produits alimentaires }\end{array}$ \\
\hline Moyen d'extinction & $\begin{array}{l}\text { Agent d'extinction gazeux } \\
\text { sur des sites à haut risque d'incendie }\end{array}$ \\
\hline $\begin{array}{l}\text { Fermentations } \\
\text { alcooliques } \\
\text { ou lactiques }\end{array}$ & $\begin{array}{l}\text { Production de boissons alcooliques } \\
\text { ou de produits de boulangerie, } \\
\text { ensilage, stockage en cave ou cale } \\
\text { de bateau, cuves de vendanges, } \\
\text { fosses, égouts }\end{array}$ \\
\hline Sources telluriques & $\begin{array}{l}\text { Grottes et cavernes, mines, volcans } \\
\text { et lacs volcaniques, émissions } \\
\text { géothermiques de gaz }\end{array}$ \\
\hline $\begin{array}{l}\text { Production endogène } \\
\text { en milieu confiné }\end{array}$ & $\begin{array}{l}\text { Scaphandriers, sous-marins, chars } \\
\text { d'assaut, abris antiatomiques, } \\
\text { stations spatiales }\end{array}$ \\
\hline $\begin{array}{l}\text { Domaine médical } \\
\text { et recherche } \\
\text { scientifique }\end{array}$ & $\begin{array}{l}\text { Cœlioscopie } \\
\text { Euthanasie animale } \\
\text { Utilisation expérimentale } \\
\text { comme agent anxiogène }\end{array}$ \\
\hline Divers & $\begin{array}{l}\text { Extraction de l'aluminium } \\
\text { par électrolyse } \\
\text { Incendies et phénomène } \\
\text { de combustion }\end{array}$ \\
\hline
\end{tabular}

chars d'assaut ou autres abris hermétiques comme les abris antiatomiques ou les stations spatiales.

Le profil type de la victime intoxiquée par du $\mathrm{CO}_{2}$ implique généralement une personne peu expérimentée, mal informée et peu habituée aux dangers potentiels du site ou de l'activité pratiquée. L'accident survient le plus souvent dans un espace confiné, mal ventilé. On peut citer à ce titre le jeune apprenti qui va nettoyer un silo, le stagiaire qui va chercher des produits dans une chambre froide défectueuse et refroidie par de la neige carbonique ou encore le touriste travaillant occasionnellement au nettoyage de cuves pour les vendanges. Ajoutons enfin l'utilisation anecdotique du $\mathrm{CO}_{2}$ comme méthode de suicide, véritable défi pour le légiste, souvent élucidé uniquement grâce à une vision globale de la scène et une enquête approfondie sur l'activité professionnelle et les connaissances chimiques du défunt (cf. infra) [15].

\section{Mesures d'urgence en préhospitalier}

Au vu des propriétés du gaz et la relative rareté des cas, la méconnaissance et la sous-estimation du risque d'une éventuelle intoxication au $\mathrm{CO}_{2}$ peuvent s'avérer fatales pour les sauveteurs. L'identification initiale se fonde essentiellement sur des indices circonstanciels (contexte professionnel) et sur l'analyse de la scène (environnement confiné, production de $\mathrm{CO}_{2}$ possible) [5]. L'utilisation d'un détecteur de $\mathrm{CO}_{2}$ est capitale pour confirmer le diagnostic et évaluer la dangerosité des lieux. Toutefois, ce type de dispositif n'est généralement pas disponible dans la phase initiale, en l'absence d'équipement spécifique des sapeurs-pompiers. Le principe de sécurité prime dès lors dans tous les cas pour les premiers intervenants.

À l'instar des intoxications au CO, l'aération des lieux et l'extraction des victimes sont des éléments clés de la prise en charge [5]. Il n'existe aucun antidote ni traitement spécifique. L'administration d'oxygène à haute concentration est recommandée dès la phase préhospitalière, bien qu'aucun consensus ne soit établi à l'heure actuelle sur la $\mathrm{FiO}_{2}$ optimale. À noter que l'effet Haldane peut théoriquement favoriser un relargage initial de $\mathrm{CO}_{2}$ dans la circulation, suite à l'administration d'oxygène, et péjorer l'intoxication. Un traitement de soutien respiratoire et hémodynamique (ventilation, amines vasoactives, intubation endotrachéale) doit 
être envisagé en fonction de la clinique : dépression respiratoire/circulatoire, trouble du rythme, crises tonicocloniques, coma, etc.

\section{Prise en charge hospitalière}

Le bilan hospitalier devra comprendre une gazométrie artérielle, une biologie sanguine complète et un ECG à la recherche de troubles ischémiques. La mesure du $\mathrm{pH}$ et des bicarbonates permet d'évaluer et de suivre l'évolution de l'acidose. Le $\mathrm{pH}$ et les bicarbonates urinaires sont à ce titre relativement simples à obtenir et sensibles aux variations du $\mathrm{CO}_{2}$ inspiré [16]. L'oxygénothérapie ainsi que les traitements de soutien devront être poursuivis. Des études animales ont évalué l'utilité d'une oxygénation hyperbare. Dans ce contexte, le traitement hyperbare paraît supérieur à une oxygénothérapie normobare pour la restauration de la fonction enzymatique, la correction du désordre électrolytique et la réduction des dommages pulmonaires, mais ne présente pas d'avantage sur l'évolution clinique, la correction du $\mathrm{pH}$, de la $\mathrm{PaO}_{2}$ ou de la $\mathrm{PCO}_{2}$ [17]. L'oxygénothérapie hyperbare peut présenter un intérêt théorique dans la prise en charge des embolies gazeuses survenant lors de cœlioscopies avec insufflation péritonéale de $\mathrm{CO}_{2}$ [18]. Il n'existe cependant pas encore de consensus clair, et des études complémentaires sont encore nécessaires pour confirmer le bénéfice de l'oxygénothérapie hyperbare dans l'intoxication au $\mathrm{CO}_{2}$.

\section{Diagnostic en cas de décès}

L'attribution d'un décès à une intoxication par du $\mathrm{CO}_{2}$ est difficile en préhospitalier et se fonde essentiellement sur l'anamnèse et sur les investigations de police. Les éléments importants à récolter sur les lieux de l'événement comprennent le contexte professionnel (usine de production, pièce confinée, travaux/défaut connu récent ?), les caractéristiques de la/des victime(s) [ouvrier expérimenté de l'usine ou nouveau collaborateur peu informé, contexte dépressif et/ou risque suicidaire] et idéalement une mesure du taux de $\mathrm{CO}_{2}$ ambiant à l'aide d'un capteur spécifique. Aucune constatation à l'examen externe n'est pathognomonique d'une intoxication au $\mathrm{CO}_{2}$. Un œdème cérébral, un œdème pulmonaire et une hyperhémie relative des organes internes peuvent être retrouvés [15]. L'analyse précoce des gaz sanguins et du $\mathrm{pH}$ artériel oriente le diagnostic. En cas de décès, quelle qu'en soit l'origine, le taux d'oxygène chute et le $\mathrm{CO}_{2}$ s'accumule. Sur les cadavres frais, on peut encore distinguer le sang du ventricule droit, plus riche en $\mathrm{CO}_{2}$, de celui du ventricule gauche [19]. En cas d'air vicié en $\mathrm{CO}_{2}$, le ventricule gauche présenterait une concentration en $\mathrm{CO}_{2}$ supérieure [15].
L'analyse des gaz résiduels pulmonaires par spectrométrie de masse permet enfin de déterminer l'atmosphère inhalée lors de la mort du patient [20].

\section{Prévention et sécurité publique}

Dans la plupart des accidents recensés, les mesures de prévention étaient insuffisantes ou absentes. L'information et la sensibilisation au risque lié au $\mathrm{CO}_{2}$ présentent ainsi de fréquentes lacunes [8]. Cependant, depuis 1957 déjà, différents principes de précaution ont été élaborés, incluant la sensibilisation des employeurs (information) et des employés (formation), incluant l'indication du danger potentiel, la ventilation au préalable des lieux confinés ainsi que l'utilisation d'appareils respiratoires adaptés si l'exposition à une grande quantité de $\mathrm{CO}_{2}$ est inévitable [8]. Les zones à risque doivent être facilement identifiables pour les secours en cas d'intervention, et des dispositifs de détection sont recommandés. Concernant les valeurs limites d'exposition professionnelle, un taux maximum de $5000 \mathrm{ppm}$ est retenu sur huit heures de travail (soit une concentration de $0,5 \%$ ) par les différents organismes américains ou européens. En cas d'exposition aiguë, des recommandations anglaises (occupational exposure standard) ou nord-américaines (American Conference of Governmental Industrial Hygienists) préconisent de limiter l'exposition à $15000 \mathrm{ppm}$ sur dix minutes, respectivement 30000 ppm durant 15 minutes [8].

\section{Conclusion}

L'intoxication aiguë au $\mathrm{CO}_{2}$ est une cause rare mais souvent fatale d'accident, par toxicité directe et par asphyxie. Cette étiologie doit être suspectée en fonction du contexte et de l'anamnèse, étant donné les propriétés physicochimiques du $\mathrm{CO}_{2}$ qui le rendent non détectable pour l'être humain en l'absence de détecteurs spécifiques. L'identification précoce est primordiale afin de garantir la sécurité des intervenants et d'éviter le suraccident. Le traitement est principalement symptomatique, avec une oxygénothérapie large, sans évidence démontrée à ce jour d'un éventuel traitement hyperbare. Le cas clinique typique est celui d'une victime unique, victime d'une intoxication ayant lieu dans un espace confiné d'une petite entreprise non industrielle dont elle n'est pas employée. Plus rarement, l'intoxication peut concerner un nombre important de patients [21].

Conflits d'intérêts : les auteurs déclarent ne pas avoir de liens d'intérêts. 


\section{Références}

1. Pfanz H, Yüce G, Gulbay AH, Gokgoz A (2018) Deadly $\mathrm{CO}_{2}$ gases in the Plutonium of Hierapolis (Denizli, Turkey). Archaeol Anthropol Sci. https://doi.org/10.1007/s12520-018-0599-5 (Dernier accès 13 juillet 2018)

2. Bliefert C, Perraud R (2009) Chimie de l'environnement air, eau, sols, déchets. De Boeck Ed., Bruxelles, pp 110-5

3. Lumb AB (2016) Nunn's applied respiratory physiology. Elsevier Ed., Edinburgh, pp 151-68

4. Guais A, Brand G, Jacquot L, et al (2011) Toxicity of carbon dioxide: a review. Chem Res Toxicol 24:2061-70

5. Langford NJ (2005) Carbon dioxide poisoning. Toxicol Rev 24:229-35

6. Ikeda N, Takahashi H, Umetasu K, Suzuki T (1989) The course of respiration and circulation in death by carbon dioxide poisoning. Forensic Sci Int 41:93-9

7. Law J, Young M, Alexander D, et al (2017) Carbon dioxide physiological training at NASA. Aerosp Med Hum Perform 88:897-902

8. Louis F, Guez M, Le Bacle C (1999) Intoxication par inhalation de dioxyde de carbone. Documents pour le médecin du travail. Institut national de recherche et de sécurité INRS. Dossier médicotechnique N79TC74. 79:179-94

9. Scott JL, Kraemer DG, Keller RJ (2009) Occupational hazards of carbon dioxide exposure. J Chemical Health Safety 16:18-20

10. Schwartz L, Guais A, Chaumet-Riffaud P, et al (2010) Carbon dioxide is largely responsible for the acute inflammatory effects of tobacco smoke. Inhal Toxicol 22:543-51
11. Abolhassani M, Guais A, Chaumet-Riffaud P, Sasco AJ (2009) Carbon dioxide inhalation causes pulmonary inflammation. Am J Physiol Lung Cell Mol Physiol 296:L657-65

12. Permentier K, Vercammen S, Soetaert S, Schellemans C (2017) Carbon dioxide poisoning: a literature review of an often forgotten cause of intoxication in the emergency department. Intern $\mathrm{J}$ Emerg Med 10:14

13. Dunford J, Lucas J, Vent N, Clark RF, et al (2009) Asphyxiation due to dry ice in a walk-in freezer. J Emerg Med 36:353-6

14. Baxter PJ, Kapila M, Mfondu D (1989) Lake Nyos disaster, Cameroon, 1986: the medical effects of large scale emission of carbon dioxid? Br Med J 298:1437-41

15. Rupp WR, Thierauf A, Nadjem H, Vogt S (2013). Suicide by carbon dioxide. Forensic Sci Int 231:30-2

16. Tomoda A, Yamanaka S, Kawai H, et al (1995) Variation of urinary $\mathrm{pH}$ and bicarbonate concentrations of students in metropolitan and rural areas of japan. Arch Environ Health 50:457-61

17. Niu YM, Hao FT, Xue CJ, et al (2012) Study on the effect of different oxygen therapies on rats with acute carbon dioxide poisoning. Hum Exp Toxicol 31:126-33

18. McGrath B, Zimmerman J, Williams J, Parmet J (1989) Carbon dioxide embolism treated with hyperbaric oxygen. Can J Anaesth 36:586-9

19. Kettner M, Ramsthaler F, Juhnke C, Bux R (2013) A fatal case of $\mathrm{CO}_{2}$ intoxication in a fermentation tank. J Forensic Sci 58:556-8

20. Srisont S, Chirachariyavej T, Peonim AV (2009) A carbon dioxide fatality from dry ice. J Forensic Sci 54:961-2

21. Halpern P, Raskin Y, Sorkine P, Oganezov A (2004) Exposure to extremely high concentrations of carbon dioxide. Ann Emerg Med 43:196-9 